\title{
Evaluación de la metacognición sobre el estudio en estudiantes de psicología
}

Evaluation of metacognition on study in psychology students

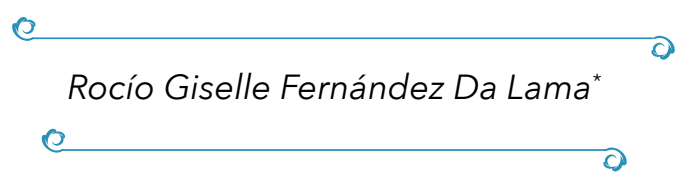

Recibido 16.05. 2019• Arbitrado 05. 06. $2019 \bullet$

Aprobado 20.06. 2019

Licenciada en Psicología, Universidad de Buenos Aires. Facultad de Psicología, Adscripta en Centro de Investigación de Psicología y Psicopedagogía, Pontifica Universidad Católica Argentina, ORCID: 0000-00031529-2926. rociogfernandezdalama@ hotmail.com

\section{Resumen}

La presente investigación evaluó la metacognición sobre el estudio en estudiantes de Psicología de la Universidad de Buenos Aires y su asociación con el rendimiento académico. Además, se investigaron diferencias en la metacognición según la edad. Se realizó un estudio no experimental, descriptivo-correlacional y transversal. Los participantes fueron 197 estudiantes universitarios (80\% mujeres y $20 \%$ hombres) entre 18 y 48 años $(\mathrm{M}=23.82$, SD = 6.14), seleccionados mediante un muestreo de conveniencia. Se administraron el Inventario de Reflexiones sobre el estudio universitario y una encuesta sociodemográfica y datos académicos. Se encontró que los estudiantes presentaron un valor promedio de metacognición en el estudio de 118.68 con una desviación de 14.30, con un puntaje máximo de 160 , distinguiendo tres puntajes parciales según el tipo de conocimiento metacognitivo, a saber, en la percepción de uno mismo $(\mathrm{M}=39.05, \mathrm{SD}=5.45$, puntaje máximo de 50), acciones específicas $(\mathrm{M}=44.35, \mathrm{SD}=$ 6.57, puntaje máximo de 60) y autocontrol en el estudio ( $\mathrm{M}=35.28, \mathrm{SD}=5.83$, puntaje máximo de 50). El análisis de los datos reveló la existencia 
de diferencias significativas según la edad en el nivel de metacognición. No se encontró asociación con el rendimiento académico. Se espera que la información reportada en esta investigación sea de utilidad a docentes, psicólogos, y demás profesionales del ámbito educativo, en pos del desarrollo de estrategias educativas que potencien los aprendizajes y mejoren el rendimiento académico.

Palabras clave: Metacognición, estudiantes, evaluación, psicología

\section{Abstract}

The present investigation evaluated the metacognition on the study in Psychology students of the University of Buenos Aires, and its association with the academic performance. Additionally, differences in metacognition were investigated according to age. A non-experimental, descriptive-correlational, and cross-sectional study was carried out. Participants were 197 university students ( $80 \%$ women and $20 \%$ men) between 18 and 48 years of age $(M=23.82, S D=6.14)$, selected by a convenience sampling. The Inventory of Reflections on the University Study and a sociodemographic survey and academic data were administered. It was found that the students presented an average value of metacognition on the study of 118.68 with a deviation of 14.30 , with a maximum score of 160 , distinguishing three partial scores according to the type of metacognitive knowledge, namely, on the perception of oneself $(\mathrm{M}=39.05$, SD $=5.45$, maximum score of 50$)$, specific actions $(\mathrm{M}=44.35, \mathrm{SD}=6.57$, maximum score of 60$)$, and self-monitoring in the study $(\mathrm{M}=35.28, \mathrm{SD}=5.83$, maximum score of 50). The analysis of the data revealed the existence of significant differences according to age in the level of metacognition. No association was found with academic performance. The information reported in this research is expected to be useful to teachers, psychologists, and other professionals in the educational field, in pursuit of the development of educational strategies that enhance learning and improve academic performance.

Keywords: metacognition, students, evaluation, psychology.

\section{Introducción}

La educación formal de nivel superior ha sido considerada, especialmente en los últimos años, como un bien de elevado valor tanto a nivel nacional como internacional (UNESCO, 1998, 2014). Principalmente, las razones del creciente interés de distintos organismos en la calidad y el acceso a la educación, se deben al viraje de una sociedad centrada en la producción de bienes y acumulación de capital, por una sociedad del conocimiento, donde se hace 
obligatorio el recurso humano formado (Alcántara, 2006; García-Manjón y Pérez-López, 2008).

En el caso de la Argentina, el sistema educativo universitario ha registrado, por un lado, tasas altas en cuanto a la cantidad de estudiantes inscriptos a las distintas carreras ofertadas -registrando un total de 1.87 millones de estudiantes universitarios en instituciones públicas y privadas-, y valores elevados en la cantidad de universidades por habitante -existe una universidad por cada 390 mil habitantes- (Guadagni, 2017), mientras que por otro lado, se registran los niveles más bajos de graduación en comparación a otros países latinoamericanos, tales como Chile, Brasil, Colombia, y México, graduándose sólo 28 estudiantes de cada 10 mil habitantes (Braginski, 2018; Guadagni, 2018).

Ante este panorama complejo, las elecciones de estudiantes según carreras universitarias presentan claras tendencias. En universidades estatales, donde es obligatoria la aprobación del Ciclo Básico Común (C.B.C.), se impone la carrera de Medicina -con un total de 9457 estudiantes inscriptos en 2018-, siguiéndole la carrera de Psicología -acumuló un total de 5176 inscriptos- (Fernández, 2018). Sin embargo, es en este mismo tramo inicial de los estudios universitarios, el C.B.C., donde los estudiantes presentan la mayor tasa de deserción académica, así como de demora en su finalización (Fernández, 2018).

Considerando las dificultades que presentan los estudiantes universitarios -deserción académica, cambio de carrera, demora en la conclusión de estudios, bajo rendimiento, entre otras- (Corominas-Rovina, 2001; Ruiz de Miguel, 2001; Mecau, Quevedo y Tello, 2012; Rodríguez-Lagunas y Hérnandez-Vázquez, 2008; Tejedor-Tejedor y Muñoz-Repiso, 2007), es que se dibuja como problemática principal el investigar sobre los aspectos referidos al aprendizaje de los estudiantes, promoviendo el desarrollo de instrumentos con probadas evidencias de una buena calidad psicométrica y adaptación local que evalúen las características de esta población. En este sentido, uno de los constructos a evaluar que ha cobrado gran notoriedad en las últimas décadas, es el de metacognición (Bara-Soro, 2001; Ozturk, 2017). El término metacognición, acuñado por Flavell (1976), se refiere al conocimiento con el que cuenta el individuo sobre sus procesos cognitivos y el control de los mismos. De esta manera, dentro de la metacognición pueden distinguirse dos componentes, por un lado, el conocimiento de los procesos cognitivos, el cual es un conocimiento estable y verbalizable, que tiene la persona sobre sus recursos cognitivos y sus posibilidades a la hora de afrontar una situación de aprendizaje; y por el otro lado, la regulación de la cognición, la cual incluye el uso de distintas estrategias, tales como planificación, monitoreo, organización, y evaluación de los procesos y estados cognitivos y sus resultados dependiendo 
de la situación, por lo que sería un aspecto inestable en mayor medida que el primero y de carácter procedimental (Brown, 1987).

La literatura científica y académica, centrada en evaluar aspectos referidos al aprendizaje del estudiantado universitario, ha demostrado que niveles altos de metacognición se relacionan con una comprensión profunda de los contenidos impartidos en clase, mejoras en la realización de tareas (MartínezFernández, Villegas y Martínez-Torres, 2004; Ellis, Goodyear, Prosser y O'Hara, 2006), y un rendimiento académico elevado (Escanero-Marcén, Soria, Escanero-Ereza y Guerra-Sánchez, 2013; Vrugt y Oort, 2008; Young y Fry, 2008). Asimismo, debido a la estrecha relación entre metacognición, conocimiento y aprendizaje, es que su estudio ha contribuido a la comprensión de los procesos involucrados en la resolución de problemas en diferentes áreas de conocimientos (Águila, 2014; Osses-Bustingorry y Jaramillo-Mora, 2008; Sandia-Rondel, 2004).

Dentro de los distintos instrumentos actualmente vigentes que evalúan la metacognición pueden nombrarse el Inventario de Estrategias Metacognitivas (O 'Neil y Abedi, 1996), el Inventario del Conocimiento Metacognitivo de las Estrategias de Interpretación de Lectura (MARSI, Mokhtari y Reichard, 2002), y el Inventario de Habilidades Metacognitivas (MAI, Schraw y Dennison, 1994). Un instrumento de reciente desarrollo en la Argentina es el inventario Reflexiones sobre el estudio universitario (Palacios y Schinella, 2017), el cual ha registrado adecuadas propiedades psicométricas en su evaluación de nivel de conciencia metacognitiva sobre los procesos de estudio en estudiantes de la carrera de Medicina de la Universidad Nacional de La Plata.

A partir de lo expuesto es que se definen como objetivos de investigación en este trabajo, la evaluación de la metacognición sobre el estudio en una muestra de estudiantes universitarios de la carrera de Psicología de la Universidad de Buenos Aires, el determinar tanto, una posible asociación entre la metacognición sobre el estudio y el rendimiento académico, y la existencia de diferencias significativas en la metacognición sobre el estudio según la edad de los estudiantes.

\section{Método}

\section{Participantes}

Participaron del estudio 197 estudiantes (80\% varones y 20\% mujeres), de entre 18 y 48 años de edad $(M=23.82, D E=6.14)$, quienes fueron seleccionados 
mediante un muestreo intencional simple. La muestra se conformó por estudiantes de todos los años de la carrera: primer año (13.7\%); segundo año (15.7\%); tercer año (28.4\%), cuarto año $(18.8 \%)$; quinto año $(8.1 \%)$, y sexto año $(15.2 \%)$.

\section{Instrumentos}

-Inventario Reflexiones sobre el estudio universitario (Palacios y Schinella, 2017), diseñado por investigadores de la Universidad Nacional de la Plata para ser administrado a estudiantes universitarios vía online, con evidencias adecuadas de validez de constructo y de contenido, así como una consistencia interna apropiada para cada una de sus dimensiones. Este instrumento evalúa por medio de 32 ítems, con formato de respuesta Likert de cinco posiciones, ( 5 =Completamente de acuerdo; $1=$ Completamente en desacuerdo) el conocimiento que el estudiante tiene sobre la percepción de sí mismo en el estudio (total de 10 ítems), las acciones específicas sobre el estudio (total de 12 ítems), y el autoseguimiento en el estudio (total de 10 ítems). Asimismo, se le da la posibilidad al estudiante de no responder si no está seguro de su respuesta, puntuando dicha opción como cero. La puntuación de los resultados se realiza mediante la sumatoria de los puntajes parciales arrojados por los tres tipos de conocimiento ya mencionados, obteniéndose un puntaje total, que varía de un puntaje mínimo de 0 a un puntaje máximo de 160 , indicador de la metacognición sobre el estudio del evaluado. Además, el inventario cuenta con tres preguntas de respuesta abierta donde el estudiante responde sin ningún tipo de restricción, y un espacio para dejar sus observaciones. Las tres preguntas en orden son: ¿Qué autocrítica tienes como estudiante?; ¿Qué tipo de lecturas/temas/materias de la carrera te resultan más difíciles para estudiar?; ¿Qué acciones/estrategias te resultan más exitosas en el estudio de las materias universitarias?

-Encuesta sociodemográfica y académicos. Se recabó información útil para la caracterización de la muestra -sexo, edad- y datos de interés para calcular el rendimiento académico -año de inicio de estudios, total de materias aprobadas-.

\section{Procedimientos}

Se realizó la administración del instrumento de manera individual, vía online mediante la plataforma para encuestas Google Forms. Los participantes para el estudio fueron contactados por medio de la red social Facebook en grupos de estudio de distintas materias de la carrera de Psicología de la Universi- 
dad de Buenos Aires. Previamente a que el estudiante completara los datos sociodemográficos y contestara los ítems del instrumento, se le presentó un consentimiento informado, el cual debía aceptar para continuar a la siguiente hoja, explicando el carácter voluntario, no obligatorio, anónimo del estudio, y que podría cesar de responder al mismo en el momento que quisiera.

\section{Análisis de datos}

Para el análisis de los datos recabados se utilizó el paquete estadístico para Ciencias Sociales SPSS 21, calculándose la frecuencia, la Media y el Desvío Estándar de las variables para responder a los objetivos descriptivos planteados. En cuanto a los análisis de correlación y de diferencias entre grupos, se comprobaron previamente los supuestos de normalidad y homocedasticidad en las variables a evaluar. Al no cumplirse ambos supuestos para la aplicación de pruebas paramétricas, se emplean pruebas no paramétricas, tales como la correlación rho de Spearman y la prueba U de Mann-Whitnney.

\section{Resultados}

\section{Análisis descriptivos}

Con el fin de describir la metacognición sobre el estudio en la muestra evaluada, se procedió a calcular la Media y el Desvío Estándar para el puntaje total $(M=118.68 ; D E=14.30)$. Adicionalmente, se calcularon las Medias y los Desvíos pertenecientes a los tres tipos de conocimientos metacognitivos, a saber, percepción de sí mismo en el estudio $(M=39.05 ; D E=5.45)$, las acciones específicas sobre el estudio $(M=44.35 ; D E=6.57)$, y el autoseguimiento en el estudio $(M=35.28 ; D E=5.83)$. En cuanto al análisis de los ítems, puede decirse que los valores medios más altos se registraron en los ítems 21 $(M=4.68 ; D E=.69)$ y $23(M=4.46 ; D E=.92)$, los cuales aluden cada uno a acciones específicas y al autoseguimiento en el estudio, respectivamente. Mientras que el valor más bajo se registró en el ítem $17(M=2.85 ; D E=1.15)$, perteneciente a las acciones específicas sobre el estudio. En la Tabla 1 pueden apreciarse los valores medios y desvíos de los 32 ítems de la escala. Otro aspecto que se manifestó en el análisis de las respuestas fue la gran cantidad de respuestas en la categoría "Ni de acuerdo ni en desacuerdo" (puntaje 3 de respuesta) en la mayoría de las afirmaciones. 
Tabla 1. Distribución de los valores medios y desvios estándares de los items.

\begin{tabular}{|c|c|c|c|}
\hline $\begin{array}{c}\text { Tipo de } \\
\text { conocimiento }\end{array}$ & Ítem & Media & Desvío \\
\hline \multirow{10}{*}{ 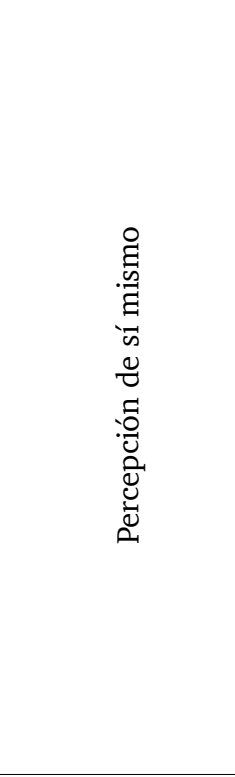 } & $\begin{array}{l}\text { 1. Me propongo metas específicas cuando estudio un } \\
\text { tema o una materia. }\end{array}$ & 3.64 & 1.34 \\
\hline & $\begin{array}{l}\text { 2. Distribuyo mi tiempo disponible para aprovecharlo } \\
\text { mejor en el estudio. }\end{array}$ & 3.75 & 1.14 \\
\hline & 3. Conozco mis puntos fuertes y débiles para estudiar. & 4.02 & 1.20 \\
\hline & 4. Sé cuán bien he actuado cuando finalizo un examen. & 3.58 & 1.05 \\
\hline & $\begin{array}{l}\text { 5. Puedo determinar el tipo de información que resulta } \\
\text { importante aprender. }\end{array}$ & 4.04 & .95 \\
\hline & $\begin{array}{l}6 \text { Soy eficiente para organizar, resumir y sintetizar la } \\
\text { información. }\end{array}$ & 3.84 & .95 \\
\hline & 7. Tengo facilidad para recordar la información. & 3.89 & .97 \\
\hline & 8. Me doy cuenta si he comprendido bien. & 4.26 & .83 \\
\hline & $\begin{array}{l}\text { 9. Soy consciente de mis estrategias/ hábitos de } \\
\text { estudio. }\end{array}$ & 4.14 & 1.01 \\
\hline & $\begin{array}{l}\text { 10. Soy capaz de resolver los problemas que se me } \\
\text { presentan en el estudio. }\end{array}$ & 3.90 & .84 \\
\hline \multirow{12}{*}{ 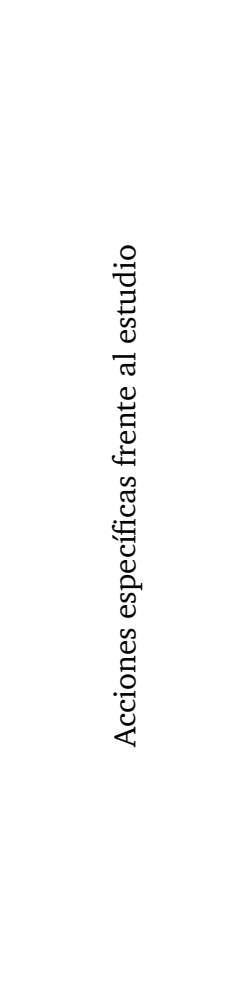 } & $\begin{array}{l}\text { 11. Pienso en todo lo que necesito, antes de comenzar } \\
\text { a estudiar. }\end{array}$ & 4.22 & .90 \\
\hline & $\begin{array}{l}\text { 12. Me detengo y reflexiono cuando encuentro } \\
\text { información importante. }\end{array}$ & 4.15 & .96 \\
\hline & $\begin{array}{l}\text { 13. Sintetizo lo que aprendí cada vez que termino de } \\
\text { estudiar. }\end{array}$ & 3.01 & 1.40 \\
\hline & 14. Pido ayuda a otros cuando no entiendo algo. & 3.40 & 1.32 \\
\hline & $\begin{array}{l}\text { 15. Centro intencionalmente la atención en la } \\
\text { información más importante. }\end{array}$ & 4.05 & .89 \\
\hline & $\begin{array}{l}\text { 16. Propongo mis propios ejemplos para mejorar la } \\
\text { comprensión. }\end{array}$ & 3.02 & 1.46 \\
\hline & $\begin{array}{l}\text { 17. Hago diagramas, cuadros, gráficos, dibujos para } \\
\text { reducir la información. }\end{array}$ & 2.85 & 1.55 \\
\hline & $\begin{array}{l}\text { 18. Trato de formular las nociones con mis propias } \\
\text { palabras. }\end{array}$ & 3.75 & 1.26 \\
\hline & $\begin{array}{l}\text { 19. Intento relacionar lo nuevo con mis saberes previos } \\
\text { sobre el tema. }\end{array}$ & 3.96 & 1.10 \\
\hline & 20. Reviso mis ideas cuando siento confusión. & 4.10 & .81 \\
\hline & $\begin{array}{l}\text { 21. Retrocedo y vuelvo a leer cuando la información no } \\
\text { me resulta clara. }\end{array}$ & 4.68 & .69 \\
\hline & 22. Organizo las tareas de estudio en distintas etapas. & 3.18 & 1.61 \\
\hline
\end{tabular}




\begin{tabular}{|c|c|c|c|}
\hline $\begin{array}{l}\text { Tipo de } \\
\text { conocimiento }\end{array}$ & Ítem & Media & Desvío \\
\hline \multirow{10}{*}{ 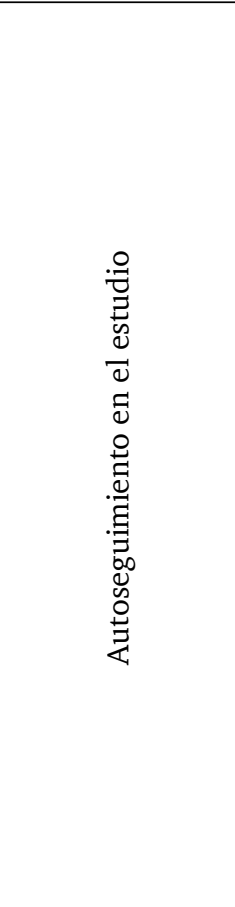 } & $\begin{array}{l}\text { 23. Trato de usar estrategias de estudio que en el } \\
\text { pasado han sido exitosas. }\end{array}$ & 4.46 & .92 \\
\hline & $\begin{array}{l}\text { 24. Empleo estrategias/técnicas de estudio con un } \\
\text { propósito específico. }\end{array}$ & 3.50 & 1.53 \\
\hline & $\begin{array}{l}\text { 25. Adecuo la forma de estudiar a las situaciones que } \\
\text { se me presentan. }\end{array}$ & 3.98 & 1.15 \\
\hline & $\begin{array}{l}\text { 26. Analizo la utilidad de las estrategias que empleo } \\
\text { mientras estoy estudiando. }\end{array}$ & 3.08 & 1.33 \\
\hline & $\begin{array}{l}\text { 27. Me pregunto si he aprendido todo lo que era } \\
\text { posible, cuando termino de estudiar. }\end{array}$ & 3.64 & 1.21 \\
\hline & $\begin{array}{l}\text { 28. Advierto cuando estoy usando estrategias de } \\
\text { estudio equivocadas. }\end{array}$ & 3.06 & 1.45 \\
\hline & $\begin{array}{l}\text { 29. Establezco pausas regulares para controlar la } \\
\text { comprensión. }\end{array}$ & 3.50 & 1.25 \\
\hline & $\begin{array}{l}\text { 30. Detecto de manera rápida cuando una estrategia de } \\
\text { estudio es la más efectiva. }\end{array}$ & 3.33 & 1.44 \\
\hline & 31. Formulo preguntas para evaluar mi aprendizaje. & 3.10 & 1.52 \\
\hline & $\begin{array}{l}\text { 32. Reviso constantemente si comprendí las relaciones } \\
\text { más importantes. }\end{array}$ & 3.62 & 1.22 \\
\hline
\end{tabular}

Fuente: Propia

\section{Análisis correlaciónales}

Para el análisis de la asociación del rendimiento académico y la metacognición, se calculó previamente el rendimiento académico mediante la estimación del cociente entre la cantidad de asignaturas aprobadas y el tiempo en años desde el momento de ingreso a la carrera hasta el momento de evaluación (De Miguel y Arias, 1999), no hallándose correlación alguna significativa (rho $=.030 ; p<.67)$.

\section{Análisis de comparación entre grupos}

Con el fin de determinar la existencia de diferencias según la edad en la metacognición sobre el estudio, se dividió a la muestra según la edad media de 23 años en dos partes: de 18 a 23 años estudiantes jóvenes y de 24 a 59 años estudiantes mayores. Luego se utilizó la prueba U de Mann-Whitnney, la cual arrojó que existen diferencias significativas $(U=3385.50 ; p<.05)$ entre el grupo de estudiantes jóvenes y el de estudiantes mayores. La Media de los estudiantes mayores en cuanto a la metacognición sobre el estudio es mayor que 
la de los estudiantes jóvenes $\left(M_{\text {mayores }}=121.65 ; D E=14.38\right.$ VS $M_{\text {jóvenes }}=117.32$; $D E=14.11)$. Asimismo, se registró una diferencia significativa según la edad en el tipo de conocimiento metacognitivo referido a las acciones específicas sobre el estudio $(U=3366 ; p<.05)$, presentando los estudiantes más jóvenes puntuaciones medias más bajas que los estudiantes mayores en este tipo de conocimiento $\left(M_{\text {mayores }}=45.85 ; D E=6.22 \mathrm{VS} M_{\text {jóvenes }}=43.65 ; D E=6.63\right)$. La información relevada puede apreciarse en una forma más detallada en la Tabla 2.

Tabla 2. Comparación de los valores medios y desvios estándares de los niveles de metacognición sobre el estudio.

\begin{tabular}{|c|c|c|c|c|c|c|}
\hline \multirow[t]{2}{*}{ Variables } & \multicolumn{2}{|c|}{$\begin{array}{c}\text { Estudiantes } \\
\text { Jóvenes } \\
\end{array}$} & \multicolumn{2}{|c|}{$\begin{array}{c}\text { Estudiantes } \\
\text { Mayores } \\
\end{array}$} & \multicolumn{2}{|c|}{$\begin{array}{c}\text { Valores } \\
\text { Estadísticos }\end{array}$} \\
\hline & Media & Desvío & Media & Desvío & $\mathbf{U}$ & $\boldsymbol{p}$ \\
\hline Percepción sobre sí mismo & 42.30 & 5.74 & 43.39 & 4.71 & 3313 & .463 \\
\hline Acciones específicas sobre el estudio & 43.65 & 6.63 & 45.85 & 6.22 & 3366 & .027 \\
\hline Autoseguimiento en el estudio & 36.91 & 5.86 & 38.35 & 3.73 & 3365.50 & .118 \\
\hline Total Metacognición sobre el estudio & 117.32 & 14.11 & 121.65 & 14.38 & 3385.50 & .031 \\
\hline
\end{tabular}

Fuente: Propia

Con respecto al análisis de las preguntas de respuesta abierta formuladas al final del instrumento, se procesó el contenido de las mismas mediante la herramienta informática denominada "nube de palabas", con el fin de identificar los términos más repetidos por los estudiantes (ver Figura 1). Los aspectos que los estudiantes mencionaron como autocrítica en mayor medida se refirieron a las dificultades para organizarse en cuanto al estudio, el contar con la lectura de textos al día, a pesar de otras responsabilidades, el dejar gran cantidad de textos para leer poco antes de los exámenes. En cuanto al tipo de lecturas/temas/materias que les resultan más difíciles, los estudiantes hicieron hincapié en las dificultades de aprender conceptos de memoria. Asimismo, establecieron que tanto las asignaturas con contenidos de neurofisiología, estadística, matemáticas, biología, e investigación, como aquellas que contienen contenido psicoanalítico, les reportaban grandes dificultades en su aprendizaje. Con respecto a las acciones/estrategias exitosas al momento de estudiar, surgieron en gran medida la confección y el uso de resúmenes, mantener la lectura de los textos al día para cada clase, resaltando la información más relevante, el aprender información de memoria, y el trabajar con otros compañeros en grupo. 

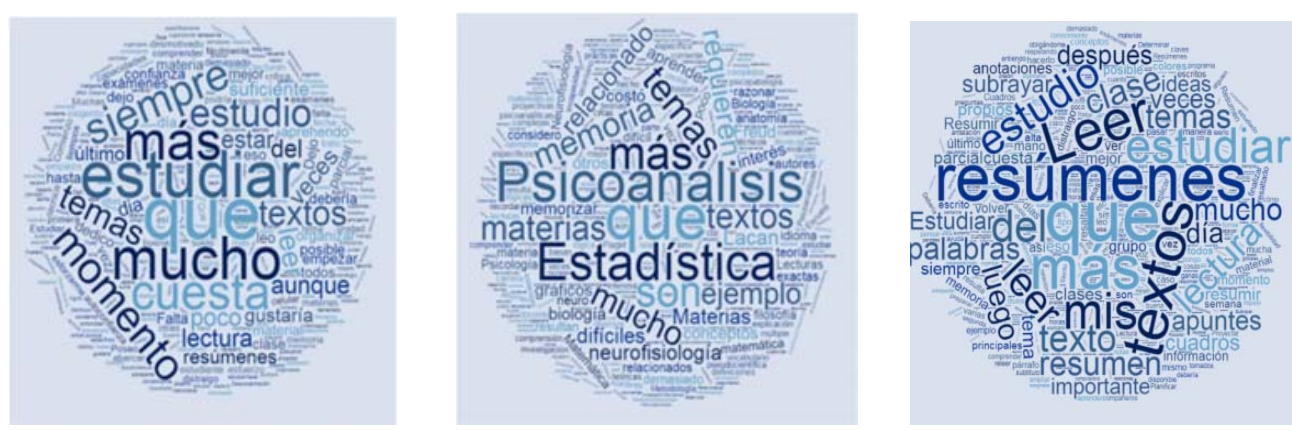

Figura 1. Nube de palabras, preguntas uno a tres ordenadas de izquierda a derecha. Pregunta 1: ¿Qué autocrítica tienes como estudiante?; pregunta 2: ¿Qué tipo de lecturas/temas/materias de la carrera te resultan más dificiles para estudiar?; pregunta 3: ¿Qué acciones/estrategias te resultan más exitosas en el estudio de las materias universitarias?

\section{Discusión}

La presente investigación evaluó la metacognición sobre el estudio en estudiantes de Psicología de la Universidad de Buenos Aires. En términos generales, los estudiantes presentaron un nivel de acuerdo (puntaje 4 en la escala) mayormente en las aseveraciones referidas a la percepción de sí mismo y a las acciones específicas frente al estudio. De este modo, los estudiantes presentaron una mayor consciencia acerca de sus puntos fuertes y débiles para estudiar, el tipo de información importante para aprender, darse cuenta si han comprendido bien, y de las estrategias/hábitos de estudio (ítems 3, 5, 8, y 9), así como también de pensar en todo lo que necesitan antes de comenzar a estudiar, detenerse y reflexionar cuando encuentran información importante, centrar la atención intencionalmente en información importante, revisar sus ideas cuando siente confusión, y retroceder y volver a leer cuando la información no resulta clara (ítems $11,12,15,20$, y 21). En cuanto a la consciencia en el autoseguimiento en el estudio, los estudiantes presentaron un mayor grado de acuerdo en el uso de estrategias de estudio que hubieran sido exitosas en el pasado (ítem 23).

En oposición a esto, los estudiantes presentaron incertidumbre al responder en la mayorías de las afirmaciones "Ni de acuerdo ni en desacuerdo" (puntaje 3 en la escala), en los distintos tipos de conocimiento, lo cual demuestra cierto desconocimiento sobre sus propios procesos de estudio. De esta manera, presentaron inseguridad en cuanto al conocimiento sobre estrategias de estudio que favorezcan la comprensión -sintetizar la información 
aprendida, proponer ejemplos para mejorar la comprensión, relacionar temas novedosos con saberes previamente aprendidos, formular preguntas para autoevaluar su proceso de aprendizaje, y revisar si comprendió las relaciones más importantes- (ítems 13, 16, 18, 19, 31, y 32), el poder determinar si sus estrategias de estudio eran adecuadas al momento de estudiar -emplear estrategias/técnicas de estudio con un propósito específico, adecuar la forma de estudiar a las situaciones que se presentan, analizar la utilidad de las estrategias empleadas mientras estudian, preguntarse si han aprendido todo lo posible al terminar de estudiar, advertir el uso de estrategias equivocadas - (ítems 24, 25, 26, 27, y 28), el manejo del tiempo -distribuir el tiempo disponible y aprovecharlo en el estudio, organizar las tareas de estudio en distintas etapas y hacer pausas regulares en la lectura de textos- (ítems 2, 22, y 29). Esto se evidenció, a su vez, en las respuestas de formato abierto, donde los estudiantes reportaron dejar la lectura de textos para días antes del parcial, así como no hacer un uso correcto del tiempo disponible en el estudio. Asimismo, los estudiantes indicaron desacuerdo (puntaje 2) en el uso de diagramas, cuadros, gráficos, dibujos para reducir la información (ítem 17). Esta información se complementa con lo respondido por los estudiantes en las preguntas de respuesta abierta, donde indicaron un mayor uso de resúmenes y el aprender información de memoria.

Otro de los objetivos de la investigación fue determinar la existencia de diferencias significativas en la metacognición según la edad. En efecto, los resultados confirman que los estudiantes jóvenes cuentan con un menor nivel de metacognición total en comparación a estudiantes de mayor edad, así como en las acciones específicas frente al estudio. Esto apunta a considerar la edad de los estudiantes como una variable a tener en cuenta a la hora de diseñar estrategias de enseñanza que posibiliten una mayor concientización sobre los procesos de estudio, especialmente las acciones y estrategias a llevar a cabo para la mejora de la comprensión y del aprendizaje en estudiantes más jóvenes o ingresantes. Estos resultados se hallan en consonancia con lo investigado por otros autores, quienes han reportado diferencias significativas en los niveles de metacognición a favor de individuos de mayor edad, tanto en población general (Justice y Dornan, 2001; Kuhn, 2000; Souchay e Isingrini, 2004; Vukman, 2005) como en estudiantes (Martínez-Fernández, 2004; Veenman y Spaans, 2004).

A la luz de los aportes de estudiosos sobre el tema, puede argüirse que la consciencia sobre el propio proceso cognitivo evoluciona con la edad y la escolarización, pasando desde los niveles más bajos -consciencia vaga y funcional- hacia niveles más elevados -consciencia reflexiva-, lo cual implica que los 
procesos metacognitivos se tornen más activos, automáticos e intencionales (e.g. Brown, 1987; Flavell, 1979; Karmiloff-Smith, 1992; González-Marqués, Suengas, y Mayor, 1993). Esta información resulta relevante al ser volcada al ámbito educativo, ya que un mayor conocimiento por parte del estudiante sobre la monitorización, regulación y ordenamiento de sus procesos cognitivos acerca de la información sobre la que operen, culminaría en un mayor conocimiento sobre sí mismo como estudiante y los factores que influyen en su rendimiento, las habilidades y estrategias de aprendizaje empleadas, y las condiciones sobre cuándo y por qué usarlas (Hofer y Pintrich, 1997; King y Kitchener, 1994; Palacios y Schinella, 2017).

Uno de los objetivos de presente trabajo era analizar la asociación entre el rendimiento académico y el nivel de metacognición sobre el estudio en la muestra evaluada, lo cual arrojó datos no significativos. Esta información se encuentra en cierta oposición a lo reportado por numerosas investigaciones (e.g. Al-Baddareen, Ghaith, y Akour, 2015; Campo, Escorcia, Moreno, y Palacio, 2016; García-Martínez, 2019; Mejía-Rodríguez, Muntada, y Cladellas-Pros, 2018), las cuales han determinado relaciones positivas entre el rendimiento y el nivel de metacognición del estudiantado. El hecho de no haber constatado dicha tendencia en el presente estudio permite, por un lado, considerar si en la carrera de Psicología, los contenidos previstos a ser estudiados y la manera en que éstos son evaluados, tienen algún tipo de influencia sobre los procesos de estudios de sus estudiantes, es decir que un mayor o menor nivel metacognitivo no representa diferencia alguna en la adquisición de conocimientos novedosos y en consecuencia en el desempeño académico; y por el otro lado, se torna necesario continuar investigando a mayor profundidad la relación entre dichas variables, especialmente teniendo en cuenta las limitaciones de este estudio.

Con respecto a las limitaciones del presente estudio, puede señalarse el tamaño reducido y la no representatividad de la muestra debido al tipo de muestreo empleado, lo cual afecta la generalización de los resultados. Otra limitación radica en el tipo de estudio realizado, ya que al tratarse de un estudio transversal, los resultados solo reflejan las características de la muestra evaluada en un momento dado, por lo que no puede tenerse en cuenta cómo éstas podrían verse modificadas a lo largo del tiempo. 


\section{Referencias}

Águila, E. (2014). Habilidades y estrategias para el desarrollo del pensamiento crítico y creativo en alumnado de la Universidad de Sonora (tesis Doctoral). Universidad de Extremadura, Badajoz, España. Recuperado de http://dehesa.unex.es/bitstream/ handle/10662/1774/TDUEX_2014_Aguila_Moreno.pdf?sequence $=1$

AL-Baddareen, G., Ghaith, S., Akour, M. (2015). Self-Efficacy, Achievement Goals, and Metacognition as Predicators of Academic Motivation. Procedia - Social and Behavioral Sciences, 191, 2068-2073. doi:10.1016/j.sbspro.2015.04.345

Alcántara A. (2006). Tendencias Mundiales en la Educación Superior: el papelde los organismos multilaterales. Revista IA Rev. Fac. Edu. UFG. 31 (1), 11-33. Recuperado de ile://C:/Users/Nancy/Downloads/BENAVIDESlaEnsenanza.pdf

Bara-Soro, P. (2001). Estrategias metacognitivas y de aprendizaje: un estudio empírico sobre el efecto de la aplicación de un programa metacognitivo, y el dominio de las estrategias de aprendizaje en estudiantes de E.S.O, B. U. P y Universidad (Tesis doctoral). Universidad Complutense de Madrid, España. Recuperado de https:// eprints.ucm.es/4765/1/T25562.pdf

Braginski, R. (2018). Informe con nuevos datos oficiales. Siente de cada 10 estudiantes no se reciben a tiempo en la universidad. Recuperado de https://www.clarin.com/ sociedad/10-estudiantes-reciben-tiempo-universidad_0_B1QH9I7gm.html

Brown, A. (1987). Metacognition, executive control, self-regulation, and other more mysterious mechanisms. En F. Weinert, y R. Kluwe (Eds.). Metacognition, motivation and understanding (pp. 65-116). New Jersey, USA: LEA.

Campo, K., Escorcia, D., Moreno, M., Palacio, J. (2016). Metacognition, writing and academic performance in Colombian and French College students. Avances en Psicología Latinoamericana, 34 (2), 233-252. doi: 10.12804/apl34.2.2016.03.

Corominas-Rovina, E. (2001). La transición a los estudios universitarios. Abandono o cambio en el primero año de universidad. Revista de Investigación Educativa, 19 (1), 1127-151. Recuperado de https://core.ac.uk/download/pdf/132556546.pdf

De Miguel, M., Arias, J. (1999). La evaluación del rendimiento inmediato en la enseñanza universitaria. Revista de Educación, 320, 353-377. Recuperado de http:// www.mecd.gob.es/dctm/revistadeducacion/articulosre320/re3201707982. pdf?documentId $=0901 \mathrm{e} 72 \mathrm{~b} 8127086 \mathrm{c}$

Ellis, R.; Goodyear, P., Prosser, M., O'Hara, A. (2006) How and what university students learn through online and face-to-face discussion: conceptions, intentions 
and approaches. Journal of Computer Assisted Learning, 22 (4), 244-256. doi: 10.1111/j.1365-2729.2006.00173.x

Escanero-Marcén, J., Soria, M., Escanero-Ereza, M., Guerra-Sánchez, M. (2013). Influencia de los estilos de aprendizaje y la metacognición en el rendimiento académico de los estudiantes de fisiología. Fundación Educación Médica, 16 (1), 23-29. doi: 10.4321/S2014-98322013000100005

Fernández, M. (06 de abril de 2018). Récord de inscriptos al CBC de la UBA: cuáles son las carreras más elegidas. Recuperado de https://www.infobae.com/ educacion/2018/04/06/record-de-inscriptos-al-cbc-de-la-uba-cuales-son-lascarreras-mas-elegidas/

Flavell, J. H. (1976). Metacognitive aspects of problem solving. En L. B. Resnick (Ed.), The nature of intelligence (pp. 231-235). New Jersey, USA: Lawrence Erlbaum.

Flavell, J. (1979). Metacognition and Cognitive Monitoring. A new área of cognitive-developmental inquiry. American Psychologist,34 (10), 906-911. Recuperado de https://pdfs.semanticscholar.org/ ee65/2f0f63ed5b0cfe0af4cb4ea76b2ecf790c8d.pdf

García-Manjón, J., Pérez-López, M. (2008). Espacio Europeo de Educación Superior, competencias profesionales y empleabilidad. Revista Iberoamericana de Educación, 46 (9), 1-12. Recuperado de file:///C:/Users/Nancy/Downloads/2444Manjon. pdf

García-Martínez, L. (2018). La autoevaluación: una alternativa constructiva para la metacognición y el rendimiento académico en un curso de Ingeniería Industrial. Educación en Ingeniería, 14 (27), 138-147. doi: 10.26507/rei.v14n27.949

González-Marqués, J., Suengas, A., Mayor, J. (1993). Estrategias Metacognitivas. Madrid, España: Editorial Síntesis.

Guadagni, A. (15 de junio de 2017). Por qué Argentina tiene más estudiantes universitarios que España. Recuperado de https://www.infobae.com/america/ opinion/2017/06/15/por-que-argentina-tiene-mas-estudiantes-universitariosque-espana/

Guadagni, A. (20 de noviembre de 2018). ¿Por qué tenemos más estudiantes universitarios pero pocos graduados? Recuperado de https://www.infobae.com/ opinion/2018/11/20/por-que-tenemos-mas-estudiantes-universitarios-peropocos-graduados/

Hofer, B., Pintrich, P. (1997). The Development of Epistemological Theories: Beliefs About Knowledge and Knowing and Their Relation to Learning. Review of Educational Research, 67 (1), 88-140. doi: 10.3102/00346543067001088 
Justice, E., Dornan, T. (2001). Metacognitive differences between traditional-age and nontraditional-age college students. Adult Education Quarterly, 51 (3), 236-249. doi: 10.1177/074171360105100305

Karmiloff-Smith, A. (1992). Learning, development, and conceptual change. Beyond modularity: A developmental perspective on cognitive science. Cambridge, USA: The MIT Press.

King, P., Kitchener, K. Wood, P. (1994). Research on the reflective judgment model. In King, P., Kitchener, K. (eds.). Developing reflective judgment: Understanding and promoting intellectual growth and critical thinking in adolescents and adults (pp. 24-202). San Francisco, USA: Jossey-Bass.

Kuhn, D. (2000). Metacognitive Development. Current Directions in Psychological Science, 9 (5), 178-181. doi:10.1111/1467-8721.00088

Martínez-Fernández, J. (2004). Concepción de aprendizaje, metacognición y cambio conceptual en estudiantes universitarios de Psicología (tesis Doctoral). Universitat de Barcelona, Barcelona, España. Recuperado de http://diposit.ub.edu/dspace/ bitstream/2445/42698/1/Tesis_final.pdf

Martínez-Fernández, J., Villegas, M., Martínez-Torres, M. (2004). Concepciones de aprendizaje y estrategias metacognitivas en universitarios venezolanos y españoles. Revista Latina de Pensamiento y Lenguaje, 12 (1), 21-35. Recuperado de http://www.redalyc.org/pdf/167/16723102.pdf

Mecau, N., Quevedo, L., Tello, L. (2012). La demora universitaria... ¿Un problema o una elección personal?. Argonautas, 2, 226-237. Recuperado de http://www. argonautas.unsl.edu.ar/files/17\%20MERCAU\%20QUEVEDO\%20TELLO\%20PDF. pdf

Mejía-Rodríguez, G., Muntada, M., Cladellas-Pros, R. (2018). Relación del funcionamiento Ejecutivo y Procesos Metacognitivos con el Rendimiento Académico en Niños y Niñas de Primaria. Revista Complutense de Educación, 29 (4), 1059-1073. doi: 10.5209/RCED.54640

Mokhtari, K., Reichard, C. (2002). Assessing Students' Metacognitive Awareness of reading Strategies. Journal of Educational Psychology, 94 (2), 249-259. doi: 10.1037//0022-0663.94.2.249

O'Neil, H., Abedi, J. (1996). Reliability and Validity of a State Metacognitive Inventory: Potential for Alternative Assessment. The Journal of Educational Research, 89 (4), 234-245. doi:10.1080/00220671.1996.9941208 
Osses-Bustingorry, S., Jaramillo-Mora, S. (2008). Metacognición: un camino para aprender a aprender. Estudios Pedagógicas, 34 (1), 187-197. doi: 10.4067/S071807052008000100011.

Ozturk, N. (2017). Assessing Metacognition: Theory and Practices. International Journal of Assessment Tools in Education, 4 (2), 134-148. doi: 10.21449/ ijate.298299

Palacios, A., Schinella, G. (2017). Diseño y validación de un instrumento para evaluar la metacognición sobre el estudio en estudiantes de Medicina. Revista de Educación de la Universidad de Granada, 24, 9-28. doi: 10.30827/reugra.v24i0.70

Rodríguez-Lagunas, J., Hérnandez-Vázquez, J. (2008). La deserción escolar universitaria en México. La experiencia de la Universidad Autónoma Metropolitana. Revista Electrónica “Actualidades Investigativas en Educación”, 8 (1), 1-30.doi: 10.15517/AIE.V8I1.9308

Ruiz de Miguel, C. (2001). Factores familiares vinculados al bajo rendimiento. Revista Complutense de Educación, 12 (1), 81-113. Recuperado de https://revistas.ucm. es/index.php/RCED/article/view/RCED0101120081A/16850

Schraw, G., Dennison, R. S. (1994). Assessing Metacognitive Awareness. Contemporary Educational Psychology, 19 (4), 460-475. doi:10.1006/ceps.1994.1033

Souchay, C., Isingrini, M. (2004). Age related differences in metacognitive control: role of executive functioning. Brain and Cognition, 56 (1), 89-99. doi: 10.1016/j. bandc.2004.06.002

Tejedor-Tejedor, F., Muñoz-Repiso, A. (2007). Causas del bajo rendimiento del estudiante universitario (en opinión de los profesores y alumnos). Propuestas de mejora en el marco del EEES. Revista de Educación, 324, 443-473. Recuperado de https://dialnet.unirioja.es/servlet/articulo? codigo $=2254218$

UNESCO. (1998). Declaración mundial sobre la educación superior en el siglo XXI: una visión y acción. Recuperado de http://www.unesco.org/education/educprog/ wche/declaration_spa.htm

UNESCO. (2014). Enseñanza y aprendizaje: lograr la igualdad para todos. Informe de seguimiento de la EPT en el mundo. Recuperado de http://unesdoc.unesco.org/ images/0022/002261/226159s.pdf

Veeman, M., Spaans, M. (2005). Relation between Intellectual and Metacognitive Skills: age and Task Differences. Learning and Individual Differences, 15, 159-176. doi: 10.1016/j.lindif.2004.12.001 
Vukman, K. (2005). Developmental differences in metacognition and their connections with cognitive development in adulthood. Journal of Adult Development, 12(4), 211-221. doi: 10.1007/s10804-005-7089-6

Vrugt, A., Oort, F. (2008). Metacognition, achievement goals, study strategies and academic achievement: Pathways to achievement. Metacognition and Learning, 3 (2), 123-146. doi.org/10.1007/s11409-008-9022-4

Young, A., Fry, J. (2008). Metacognitive awareness and academic achievement in college students. Journal of Scholarship of Teaching and Learning, 8 (2), 1-10. Recuperado de https://scholarworks.iu.edu/journals/index.php/josotl/article/ view/1696 